OITS-541

April 1994

\title{
CLUSTER GROWTH IN TWO-DIMENSIONAL QUARK-HADRON PHASE TRANSITION
}

\author{
Rudolph C. Hwa ${ }^{1}$ and Jicai Pan ${ }^{2}$ \\ ${ }^{1}$ Institute of Theoretical Science and Department of Physics \\ University of Oregon, Eugene, Oregon 97403 \\ ${ }^{2}$ Department of Physics, McGill University \\ Montreal, Quebec, Canada H3A 2T8
}

\begin{abstract}
The problem of hadronic cluster production in heavy-ion collisions is studied in search for an observable signature of first-order quark-hadron phase transition. The study is carried out by cellular automata in a two-dimensional model of the mixed phase at midrapidity. The clusters are allowed to grow as well as to coalesce upon collision. The distribution of cluster sizes is found to exhibit scaling behavior that is independent of the size of the mixed region, nucleation radius and nucleation probability. The universal scaling index $\gamma=1.86 \pm 0.18$ may be used to characterize and identify the phase transition process. Possible connection with self-organized criticality is pointed out.
\end{abstract}




\section{INTRODUCTION}

In a recent paper we have initiated the study of cluster production in quarkhadron phase transition in heavy-ion collisions [1]. The purpose is to investigate the possibility of the existence of new and interesting features that characterize the first-order phase transition that are independent of the details of the hadronization process, which may be so complicated as to render any feasible analytical approach too oversimplified. We used a cellular automaton to show the existence of a scaling behavior in the sizes of the hadronic clusters produced. Although the study is susceptible to the criticism in that the rules adopted are also an oversimplication of the phase transition process, the focus has been shifted from finding an analytical description of the problem on the basis of ignoring certain complications to focusing on those complications in the framework of some simple rules. Specifically, our emphasis has been on the nature of fluctuations of the cluster sizes by taking into account cluster collisions, coalescence and breakup during the growth process. In [1] we consider only the 1-dimensional problem, which is a drastic simplification of the realistic case. In this paper we make the first generalization to two dimensions, and examine the associated complexity. We shall show the persistence of the scaling behavior and investigate the origin of the phenomenon.

We mentioned in [1] the possible connection between the cluster growth problem and self-organized criticality (SOC) [2]. If SOC is defined as the behavior of an extended system that exhibits nontrivial scaling properties without the tuning of a parameter, then we have found that under certain general conditions the hadronization of a quark-gluon plasma possesses the characteristics of SOC. However, it is not clear what the nature of the critical point is. It is not an alternative, nonthermal description of a system going through what is usually regarded as a first-order phase transition of a thermal system. We have not understood the phenomenon well enough to connect the critical behavior to some features in continuum mechanics, such as a singularity in the diffusion constant for the sandpile problem [3]. What we have found are some interesting properties of heavy-ion collisions that are worthy of more extensive examination from various points of view.

In two dimensions (2D) there are new features that are absent in 1D. The most prominent one is that the clusters can have various shapes. Depending on the detail dynamics, such as the positivity and magnitude of surface tension, different types of shapes may develop as the clusters grow. The probabilities of coalescence and 
breakup will be affected; they in turn can influence the cluster shapes. Clearly, the more dynamical features are to be included in the modeling, the more complicated the rules of the cellular automaton will have to be. The problem will then become one opposite in approach to what are usually done in simulations for SOC, the aim of which is to adopt the simplest set of rules that can generate a phenomenon that exhibits the SOC behavior. For example, for the sandpile problem [2] or the forest fire problem $[4,5]$ the models are not close to reality but are nevertheless interesting because each of them exemplifies a class of problems that breaks new ground in statistical physics. Our problem here is somewhere in between. We are interested in a specific problem: that is, the discovery of observable signatures of quark-hadron phase transition in heavy-ion collisions. The relevant dynamics that controls the nature of fluctuations at the transition point is not known well enough to specify the rules of cluster growth. Indeed, the aim is to search for possibly interesting features that are independent of the details of the rules, and if those features exist in the modeling, then there will be strong motivation to look for them in the experiments. What are interesting about fluctuations are scaling and universal properties, which should emerge from simple models, not complicated ones, if the results are to be taken seriously as being generic. Thus we must, at least in the beginning, try to be as simple as possible, while maintaining as much as possible the essential ingredients of the growth process that characterize the heavy-ion collisions.

In the following we shall for the sake of simplicity ignore the possibility of breakup of the clusters on their way out of the plasma. In the $2 \mathrm{D}$ case it is possible to devise many ways to break up a cluster, with or without collisions. After examining a few possibilities, we have decided to leave out breakup altogether, in order not to confuse the issue of what is responsible for the results obtained without breakup.

In a realistic collision problem there are many parameters that specify the system undergoing phase transition, e.g., size of the mixed phase, transverse expansion velocity, critical radius for nucleation, nucleation rate, growth rate, etc. Varying all those parameters will complicate the problem so much that the essential characteristics of the scaling behavior will easily get lost. We shall therefore vary a few parameters only to see the sensitivity of the result. We emphasize that those parameters are not varied in order to bring the system to the critical point, but for the purpose of exploring the universality of the result. That is a very important difference. 


\section{CLUSTER FORMATION}

To pose the physical problem we adopt the conventional global picture of the collision of two high- $A$ nuclei at very high energy. After impact there is rapid longitudinal expansion with slower radial expansion. Focusing on a thin slice of the expanding cylinder at midrapidity, we have a disk with higher temperature in the interior surrounded by an annular ring in which the plasma is in the mixed phase at $T_{c}$, referred to as the $M$ region. Hadrons and hadronic clusters are formed in the $M$ region; the hotter plasma in the interior feeds the $M$ region with quarks and keeps it at $T_{c}$. The interior region shrinks as the temperature is lowered in time, and the $M$ region shrinks as the hadronic clusters are emitted from the boundary, even though quarks and hadronic clusters all move radially outward.

Restricting our attention to only the $M$ region, we map the $2 \mathrm{D}$ annular ring to a 2D square lattice of size $L \times L$ initially. We impose periodic boundary condition in the vertical $y$ direction, and regard the first column at $x=1$ as the inner boundary of the ring, and $x=x_{y}(t)$ as the outer boundary (depending on $y$ ) that changes with time $t$, starting with $x_{y}(0)=L$ for all $y$. The dependence of $x_{y}(t)$ on t will be a result of the process of cluster emission from the plasma and a smoothing process to be described below.

Since we attempt to model the phase transition process as realistically as possible, but without knowing all the dynamical mechanisms from first principles, our rules will be elaborate by the standards of SOC, yet rather simple from the physical point of view. Instead of writing down a set of differential equations in the continuum limit that completely specify the dynamics, which we know very little, we describe the cluster formation process step-by-step in a series of rules. These rules are presented here as a starting point, but can be modified as the description of the process is varied or improved.

(a) Space-time. The spatial coordinates $(x, y)$ are discretized into $L \times L$ units initially. We continue to refer to the lattice space as the $M$ region, which will change in size with time. In each time step all the subprocesses to be described below are completed before another step is taken. However, since the subprocesses involve mainly near-neighbor movements, no assumption of infinitely slow evolution rate is assumed.

(b) Single-site nucleation. Initially, all sites are unoccupied, representing quark phase. Let each site have the probability $p$ of being occupied in each time step, 
representing a local hadron phase. The initial hadron (not yet the type to be detected later, since it is at $T_{c}$ ) is thus of size $S_{0}$, which is slightly less than the intersite distance, so that neighboring sites can both be newly occupied without forming a cluster. In brief, we denote single-site nucleation ( $\mathrm{SSN})$ as $S_{0}=1$. At the next time step only the unoccupied sites can become occupied with probability $p$ at each site again.

(c) Growth. If a newly occupied site is a nearest neighbor of an old occupied site, we regard it as a growth process, and require the two to be bonded to form a cluster (or an extension of a cluster, if the old site is already part of an existing cluster). All sites in a cluster are bonded. Two neighboring sites need not be bonded except in this growth process; in particular, two newly nucleated sites by themselves are not bonded, as stated in (b) above.

(d) Average drift. All hadrons drift toward the outer boundary at a constant velocity, simulating the radial expansion. This drift motion in the $M$ region is represented by the requirement that all occupied sites $s_{i}(t)=\left(x_{i}, y_{i}\right)$ are to move to $s_{i}(t+1)=\left(x_{i}+1, y_{i}\right)$ at the next time step, assuming no fluctuations. A vacated sites becomes unoccupied.

(e) Random walk. Regarding the clusters as massive colloids in a fluid of the quarkgluon plasma, we require that they take random walks around their average drift as in Brownian motion. Thus if an occupied site in a cluster is at $\left(x_{i}, y_{i}\right)$, it is moved at the next time step to one of the following four possible sites, $\left(x_{i}+2, y_{i}\right),\left(x_{i}+1, y_{i}+1\right)$, $\left(x_{i}+1, y_{i}-1\right)$, and $\left(x_{i}, y_{i}\right)$, with equal probability. The average is $\left(x_{i}+1, y_{i}\right)$ as stated in (d). All the other sites in the cluster move together as a rigid body.

(f) Coalescence. As the clusters move, they may collide. A collision occurs during the random walk when there is an overlap of occupied sites belonging to two different clusters. When that happens, we require that the two clusters are treated as one thereafter, with one of the double at the overlap site be taken to occupy the nearest unoccupied site (at random if more than one), and be bonded to the rest of the enlarged cluster.

(g) Boundaries. Since the quark phase on the inside of the annular ring supplies the quarks to the mixed region, we require that the quark density in the $M$ region remains constant throughout, including the boundary at $x=1$. That means that all unoccupied sites remain unoccupied until an occupied site moves there, or a 
nucleation takes place there. Unoccupied sites do not drift. At the outer boundary hadron clusters can leave the $M$ region, but not the quarks due to confinement. Let a perimeter site be defined to be the site to the immediate right of the site in the $M$ region with the largest $x$ value for every fixed $y$, the latter being either occupied or unoccupied. When a cluster of $S$ sites breaks through the outer boundary, defined by any of the $S$ sites occupying a perimeter site, then the whole cluster is removed from the $M$ region, which is now redefined to have $S$ sites less, having a transient boundary.

(h) Smoothing the outer boundary. By removing $S$ sites from the $M$ region, the transient boundary can become very irregular due to the "hole" created. Assuming positive surface tension, we smooth the transient boundary by filling the hole with nearby unoccupied sites until a vertical boundary is achieved at the location of the hole. It cannot extend to the entire height $L$, since it would depend on the value of $S$.

Obviously, if $S$ is small compared to $L$, there will be an indentation of only $S$ sites one column to the left of the previous boundary line. If $S$ is not small, then filling the hole with $S$ unoccupied sites may expose other occupied sites near the boundary, in which case the affected cluster must also be emitted, resulting in further smoothing. This should be continued until all existing clusters are within the new real boundary, before another time step is taken.

(i) Evolution. After all clusters have taken their walks, have enlarged due to possible coalescence, and have been removed from the $M$ region upon crossing the outer boundary, which is then smoothened, we initiate another round of nucleation at the remaining unoccupied sites with the same probability $p$. If it occurs at a site next to an existing cluster, it is to be bonded to that cluster as described in (c). Then we let each cluster take a step in random walk and repeat the process, again and again until all clusters are emitted from the plasma. The evolution stops when there are no more unoccupied sites left, and the phase transition is over.

(j) Cluster-size distribution. With $n_{j}(S)$ denoting the number of clusters of size $S$ emitted in the $j$ th event, and $N_{j}=\sum_{S} n_{j}(S)$, we define the average distribution after $\mathcal{N}_{\text {evt }}$ events by

$$
P(S)=\frac{1}{\mathcal{N}_{\text {evt }}} \sum_{j=1}^{\mathcal{N}_{\text {evt }}} \frac{n_{j}(S)}{N_{j}} .
$$


Our model calculation of cluster size $S$ may or may not coincide with the experimental determination of $S$, since a cluster at the boundary of the plasma is at $T_{c}$, while the detector measures individual hadrons in $T=0$ vacuum. It is an issue that is outside the scope of the present investigation. However, it is not unreasonable to expect that if there is a scaling behavior in $P(S)$, the scaling index may be identical in the two situations. In any case, (1) should be used for the experimental definition of $P(S)$, whatever algorithm is used for the identification of a cluster.

The above rules form the simplest set that can adequately describe the $2 \mathrm{D}$ problem at hand. Note that there is no breakup, and that coalescence occurs with $100 \%$ probability when two clusters collide. Thus there are no parameters to adjust that affect the nature of the outcome of a collision. The only obvious parameter in the problem is $p$, which summarizes all the dynamics of nucleation. It is, however, not under experimental control, so it cannot be used to tune the system to the critical point. For every $p$ we shall carry out the simulation and calculate $P(S)$, and then vary $p$ to check the dependence of the result on $p$. Ordinarily, the lattice size $L$ is not a parameter in a lattice calculation in the sense that one usually chooses $L$ to be as large as possible, given one's computer capability. But since our initial nucleation size $S_{0}$, which has a physical meaning, is set equal to one unit of lattice spacing, $L$ is therefore the initial size of the $M$ region in units of $S_{0}$, and cannot be arbitrary. Results of the calculation for different values of $L$ correspond to different physical conditions in the heavy-ion collision.

Based on the above rules we have performed the calculation for $L=16$ for various values of $p$. The results after $10^{3}$ events of simulation with $p$ ranging from 0.01 to 0.5 are shown in Fig. 1. Evidently, there is a scaling behavior

$$
P(S) \propto S^{-\gamma}
$$

for $p \geq 0.05$. For smaller values of $p$ there is not enough time for the clusters to grow to large sizes to exhibit a scaling behavior. This is a finite-size effect for such low nucleation rates only. For higher $p$ not only can the clusters grow faster, but also there are more clusters around to enhance the collision frequency. The straightline behavior in the log-log plot then becomes more manifest, and the slope $\gamma$ is clearly identifiable.

The finite-size effect can be checked by increasing $L$. In Fig. 2 the results for $L=32$ are shown for the same set of $p$ values. The linear regions are clearly 
extended for all $p$. Thus given enough time for growth all cases exhibit scaling property. Since no parameters have been tuned to bring the system to exhibit that behavior, this result may be regarded as evidence for SOC.

To see the dependence of $\gamma$ on $L$, we show in Fig. 3 a comparison of the two cases $L=16$ and 32 for $p=0.2$. In the linear portion the two distributions essentially overlap. The corresponding slope gives

$$
\gamma=1.85 .
$$

Thus there is independence on $L$, which means independence on the initial size of the $M$ region. That is of physical signficance, since it implies that under different collision conditions there is universality in the value of the scaling index $\gamma$. From Figs. 1 and 2 one can see that this independence on $L$ is valid also for other values of $p$.

The dependence of $\gamma$ on $p$ is also not significant, although not entirely negligible. For $p$ ranging over a factor of 10 , from 0.05 to 0.5 , the value of $\gamma$ changes from 2.04 to 1.68 , which is a $10 \%$ deviation from the mean value, i.e.,

$$
\gamma=1.86 \pm 0.18
$$

Thus within that accuracy we may regard $\gamma$ as being independent of $p$. In that context we may state that the scaling behavior obtained so far is broadly universal, independent not only of the conditions under which the quark-gluon plasma is formed, but also of the dynamics of nucleation parametrized by $p$. That is, of course, of great phenomenological significance, since it would allow experimental verification of the property found here with few restrictions.

It is of interest to ask what happens in the limit of large $p$. If $p=1$, all sites would be occupied in the first step. One might then think that it would lead to one large cluster, and $\gamma$ would become a large negative number. However, that is not the case because neighboring occupied sites are not bonded until they overlap. While large clusters are in the process of being formed by successive overlaps, some of the low $S$ clusters will have left the $M$ region and populate the low $S$ portion of the $P(S)$ distribution. In Fig. 4 we show the result for $p=0.9$ for $L=16$ and 32 . One first notices that there is no increase at large $S$. Roughly, they follow the same trend as for lower $p$, but there is no linear region and the two distributions do not 
overlap. For $L=16$ the curve is imbedded in the range covered by the curves in Fig. 1, but for $L=32$ and $S>2, P(S)$ lies above all the curves in Fig. 2. It suggests that for such a large $p$ large clusters can form if given enough time, but sufficient numbers of small clusters are always emitted in the early phase of the transition to yield a distribution that is not too far off from the scaling result of Figs. 1 and 2. We shall not consider these unphysically large values of $p$ again in the following.

\section{DOUBLE-SITE NUCLEATION}

There is one more aspect of the cluster formation problem that we have not varied, viz., the critical radius of nucleation. In the previous section we considered single-site nucleation (SSN), which is equivalent to setting $S_{0}$ at essentially one unit of lattice spacing. Now let us consider a larger nucleation radius without changing anything else. We do it by requiring double-site nucleation (DSN), which turns out to necessitate some change of rules.

We replace rule (b) by

(b') Double-site nucleation. Let each unoccupied site still have probability $p$ of being occupied in each time step, but if it happens, its occupancy is to be regarded as virtual. When two virtual sites are nearest neighbors, then they become two bonded real sites within the same time step. It is a newly created hadron. Three or more virtual sites in an aggregate are bonded in pairs only as new hadrons next to one another, but not as a big cluster. The odd one left out after pairing (done randomly) is discarded. A single virtual site that is a near-neighbor to an existing cluster is bonded to that cluster in a growth process, as in (c). However, if a single virtual site stands alone in an environment of unoccupied sites, then nucleation fails, and we evict the occupant and declare the site unoccupied.

This rule simulates the failure of a hadronic bubble with radius less than the critical radius to realize its hadronization. Our initial hadron is a two-site "cluster", whose shape should not be taken to have any physical significance (e.g., a colorsinglet bound-state); it is merely the simplest nontrivial configuration. With this rule the simulation can proceed as before. Note that nucleation now takes place with probability $p^{2}$, while growth of an existing cluster at a neighboring site can take place with probabililty $p$. This is more in accord with the physical reality that growth is easier than nucleation. 
There is a small problem when the evolution is near the very end. If there is an isolated unoccupied site in the first column at the inner boundary, it can never become occupied, and the hadronization process will never be completed. Thus we must add one more rule.

(k) Contracting the inner boundary. Whenever an unoccupied site at $x=1$ along the inner boundary becomes isolated (without occupied or unoccupied sites as neighbors), it must be moved to the nearest site that is next to another unoccupied site, before another time step is taken. If it is the last unoccupied site, it is discarded.

This rule effectively eliminates some nonsites from interferring with the nucleation process. Instead of being arbitrary, it actually mimics reality to a certain extent. The mapping of the annular ring (that becomes a disk near the end) to a square lattice becomes singular when the inner quark phase shrinks to zero, so removing the nonsites from our consideration softens that singularity somewhat.

The produced clusters can be measured in terms of their cluster sizes $S$, as before. However, since the initial size $S_{0}$ is now doubled, we can consider a normalized cluster size, $S^{\prime}$, measured in units of $S_{0}$ :

$$
S^{\prime}=S / 2 .
$$

It is the comparison of $P\left(S^{\prime}\right)$ in this case (DSN) with $P(S)$ in the previous case (SSN) that is meaningful, since the growth of a cluster size should be measured relative to its initial size. In the following we shall always use the normalized size $S^{\prime}$, but with the prime omitted, so only $S$ will appear in its place. It means that there will be half-odd-integer values of $S$. The normalization of $P(S)$ remains to be

$$
\sum_{S} P(S)=1
$$

where the sum over $S$ now runs over integers and half-odd integers.

In Figs. 5 and 6 are shown our results for DSN $\left(S_{0}=2\right)$ for $L=16$ and 32, respectively. Their general features are similar to those shown in Figs. 1 and 2, except for the oscillations at small $S$ and large $p$. The latter can be understood as follows. At large $p$ there can be many DSN taking place. Their collisions with one another lead to increases in cluster sizes involving even number of sites. In the beginning growth by coalescence is more efficient than growth by bonding single 
neighbors one at a time near the outer boundary, the latter mechanism contributing to odd numbers of total sites in a cluster. That is why there is a dip at $S=1.5$ for $p=0.5$. At large enough $S$ the clusters are emitted later in the evolution, so both mechanisms will have enough chances to operate to smooth out of the distribution.

Let us ignore the oscillation and consider a line through the middle representing the average distribution. We see then that the results in Figs. 5 and 6 again show scaling for all values of $p$ except $p=0.01$, just as in the SSN case. The slopes of the linear portion again do not depend on $p$ sensitively. Fig. 7 shows the comparison of the two cases $L=16$ and 32 for $p=0.2$. The dependence on $L$ is evidently negligible in the scaling region.

To provide a better comparison of the results in the two cases, SSN and DSN, we select the $p=0.2$ distributions and exhibit them in Figs. 8 and 9 for $L=16$ and 32 , respectively. The reason why the dashed $\left(S_{0}=2\right)$ curves are lower than the solid $\left(S_{0}=1\right)$ curves is due to the normalization condition (6). For the solid curves the sum is over integer values of $S$ only, while for the dashed curves the sum must include the half-odd integers as well. The difference has no effect on the slopes. Thus again $\gamma$ is the appropriate characterization of the problem, and is now shown to be independent of the nucleation radius $S_{0}$ also.

\section{CONCLUSION}

We have investigated the problem of cluster growth in heavy-ion collisions when there is a quark-hadron phase transition, and found interesting scaling behavior in the distribution of cluster sizes that exhibit universal properties. The two-dimensional approximation of the geometry of the problem is not an unreasonable oversimplification, if one restricts one's observational domain to a small rapidity interval at midrapidity. The square lattice approximation may distort the result somewhat, but the general features of the result should remain unchanged.

We have found that for $p$ in the range from 0.05 to 0.5 the scaling index $\gamma$ is around 1.9. It is independent of the size of the $M$ region, which in turn is dependent on the collisional conditions of the heavy ions, assuming that a quark-gluon plasma is formed. Since from collision to collision the impact parameter changes and the initial temperature may vary, it is crucial that we focus on some quantity that is insensitive to those experimental conditions. It is also important that the quantity 
is insensitive to those parameters that are unknown or poorly known in theory, such as the critical nucleation radius and nucleation rate. The independence of $\gamma$ on $S_{0}$ and its approximate independence on $p$ further promote $\gamma$ as an observable that can possibly survive the experimental and theoretical uncertainties to serve as a numerical characterization of the phase transition process.

We have referred to the problem as one in quark-hadron phase transition, which carries the usual connotation that the system under study is in thermal equilibrium. Indeed, we have set up the problem in the familiar framework built on the hydrodynamical description of an expanding cylindrical fluid with a temperature profile. We then focused our attention on the mixed phase, which again is defined in the conventional framework of equilibrium thermodynamcis. However, the heart of the cellular automata does not rely crucially on the validity of those concepts. If thermal equilibrium were not achieved in heavy-ion collision, we would still have the problem of hadronization of a dense quark-gluon medium, and the rules that we have adopted to simulate what may happen may still be applicable with little modification. Thus by working with cellular automata without reference to such quantities as free energy, temperature, pressure, equation of state, etc., the validity of our investigation may transcend that of the study of quark-gluon plasma based on the usual hypotheses. If the physics involved can be further illuminated by recognizing it as a process in self-organized criticality, perhaps deeper understanding will be forthcoming from an unexpected quarter in theoretical physics.

We, of course, do not know from first principles whether our description of the cluster growth process is correct in nature. What is presented here is a first step in the direction of asking questions that cannot at the moment be easily posed in the continuum approximation of the complicated system, let alone answered. Ultimately, it is the experimental verification of what we have found here that can give credence to our description of the dynamical process. Such experimental investigations are not likely to be contemplated, if there is no focus on what to look for or hint of what might be in store. In that respect it is hoped that our finding of a universal scaling index may provide the needed impetus.

\section{Acknowledgment}

We have benefitted from helpful comments by T. Hwa on our earlier work. This work was supported in part by the U.S. Department of Energy under Grant 
No. DE-FG06-91ER40637, and by the Natural Science and Engineering Council of Canada and by the FCAR fund of the Quebec Government. 


\section{REFERENCES}

[1] R.C. Hwa, C.S. Lam, and J. Pan, Phys. Rev. Lett. 72, 820 (1994).

[2] P. Bak, C. Tang, and K. Wiesenfeld, Phys. Rev. A 38, 364 (1988).

[3] J.M. Carlson, J.T. Chayes, E.R. Grannan, and G.H. Swindle, Phys. Rev. Lett. 65, 2547 (1990).

[4] P. Bak, K. Chen, and C. Tang, Phys. Lett. A 147, 297 (1990).

[5] P. Grassberger and H. Kantz, J. Stat. Phys. 63, 685 (1991).

\section{Figure Captions}

Fig. 1. Cluster-size distribution $P(S)$ for single-site nucleation (SSN) and $L=16$, and for various values of nucleation probability $p$.

Fig. 2. $\quad$ Same as in Fig. 1 but for $L=32$.

Fig. 3. $p=0.2$ from Figs. 1 and 2 .

Fig. 4. $\quad P(S)$ for $p=0.9$.

Fig. 5. Same as in Fig. 1 but for double-site nucleation (DSN).

Fig. 6. Same as in Fig. 2 but for DSN.

Fig. 7. $p=0.2$ from Figs. 5 and 6 .

Fig. 8. $\quad p=0.2$ from Figs. 1 and 5 .

Fig. 9. $p=0.2$ from Figs. 2 and 6 . 
This figure "fig1-1.png" is available in "png" format from: http://arxiv.org/ps/hep-ph/9405268v1 
This figure "fig2-1.png" is available in "png" format from: http://arxiv.org/ps/hep-ph/9405268v1 


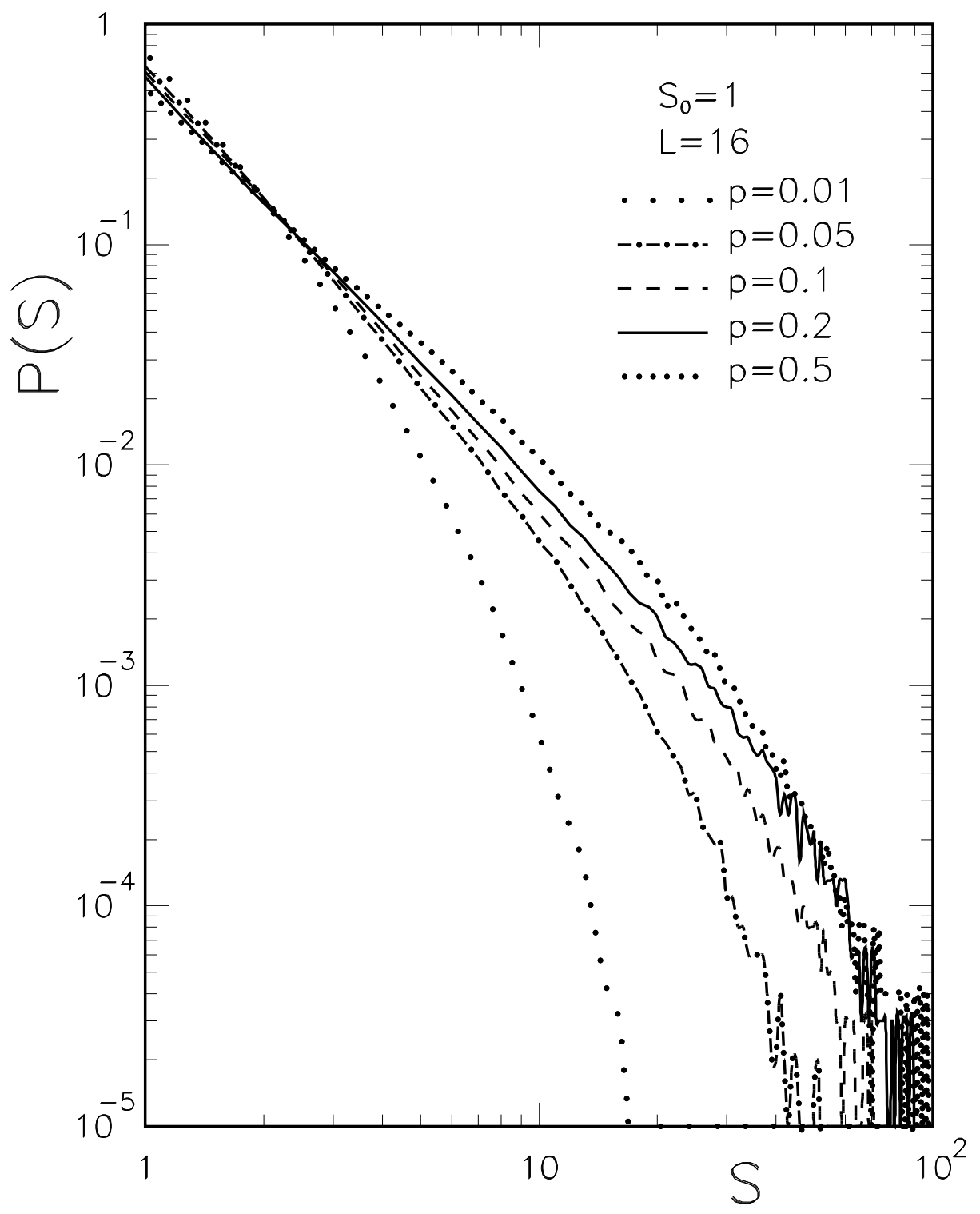

Fig. 1 
This figure "fig1-2.png" is available in "png" format from: http://arxiv.org/ps/hep-ph/9405268v1 
This figure "fig2-2.png" is available in "png" format from: http://arxiv.org/ps/hep-ph/9405268v1 


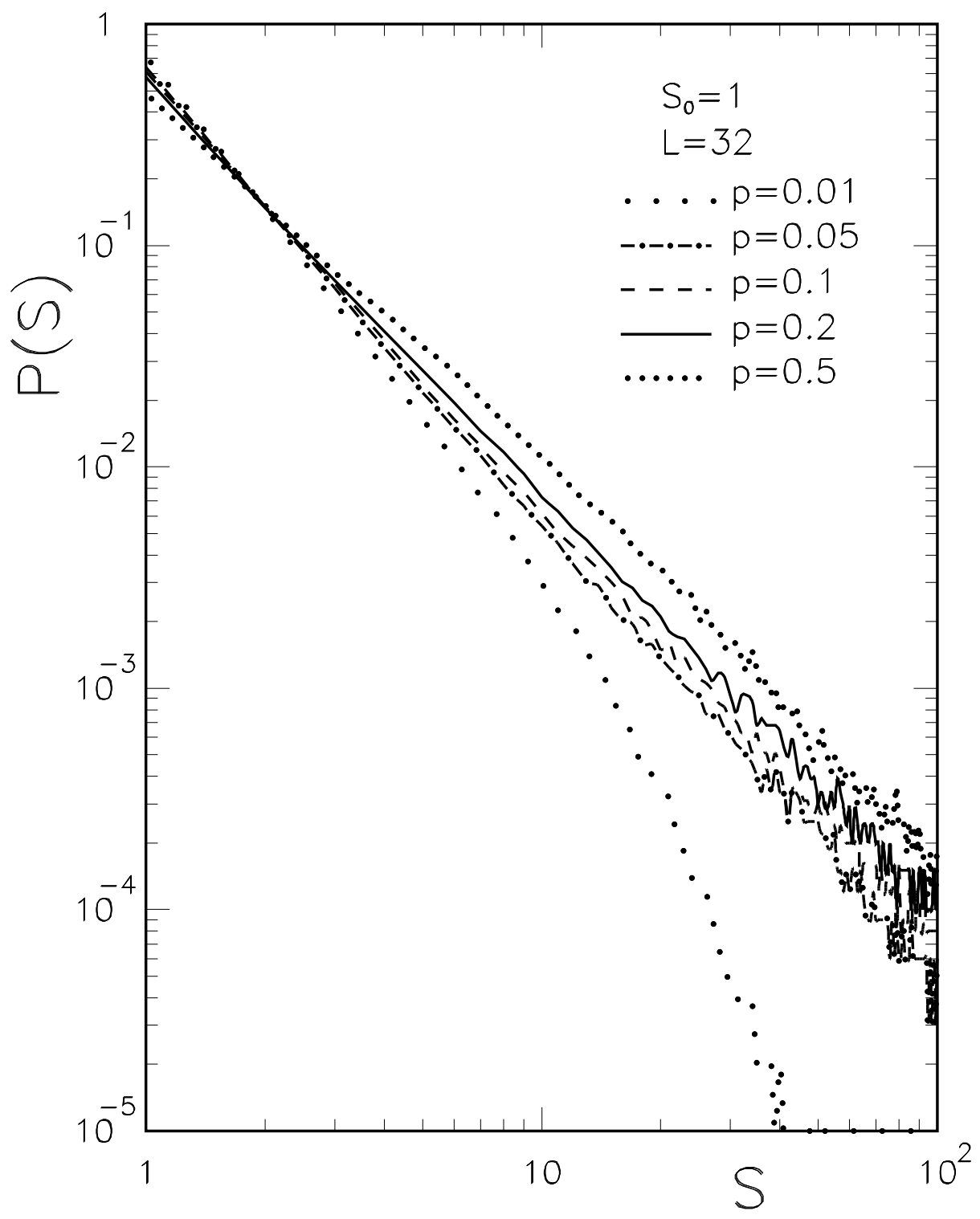

Fig. 2 
This figure "fig1-3.png" is available in "png" format from: http://arxiv.org/ps/hep-ph/9405268v1 
This figure "fig2-3.png" is available in "png" format from: http://arxiv.org/ps/hep-ph/9405268v1 


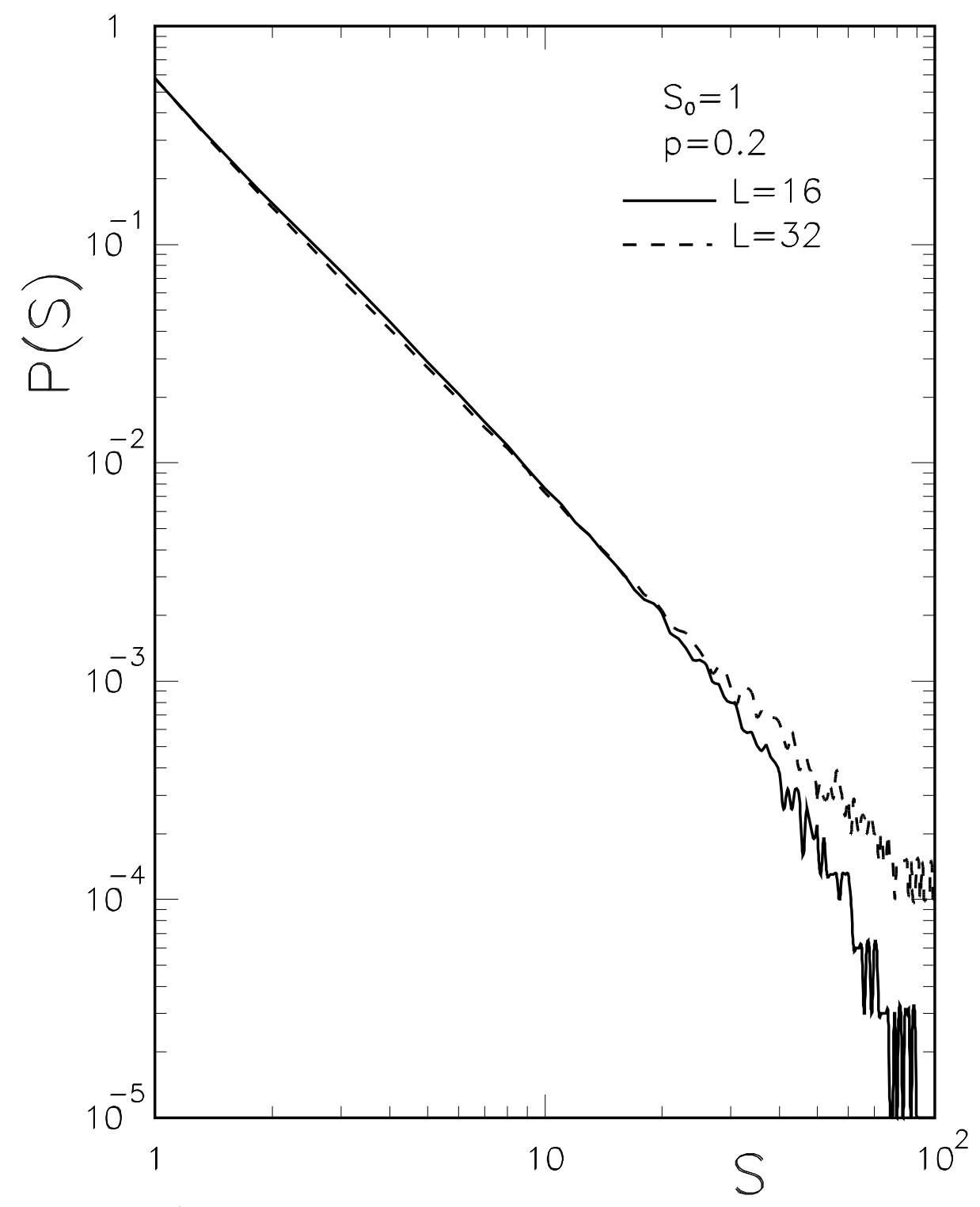

Fig. 3 
This figure "fig1-4.png" is available in "png" format from: http://arxiv.org/ps/hep-ph/9405268v1 
This figure "fig2-4.png" is available in "png" format from: http://arxiv.org/ps/hep-ph/9405268v1 


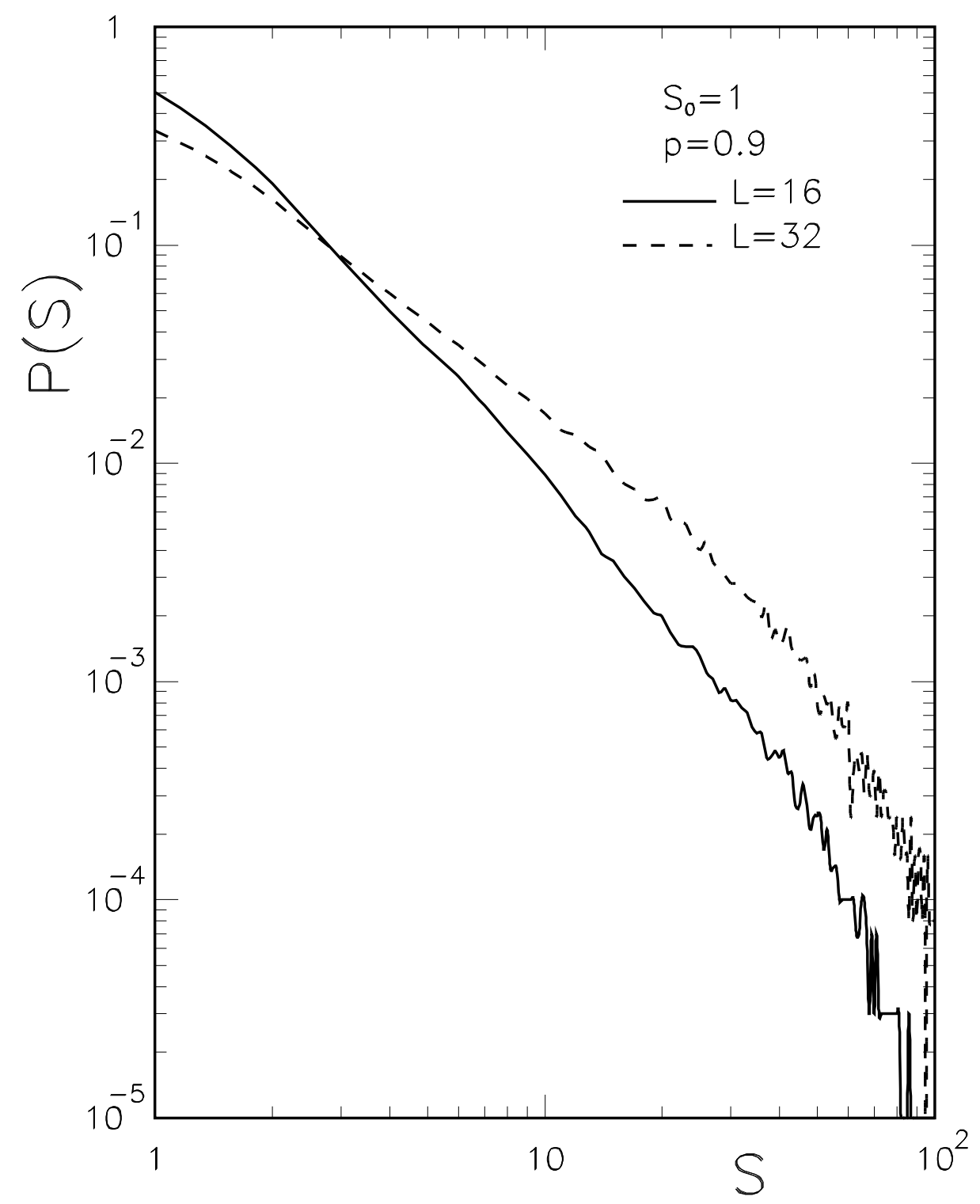

Fig. 4 
This figure "fig1-5.png" is available in "png" format from: http://arxiv.org/ps/hep-ph/9405268v1 


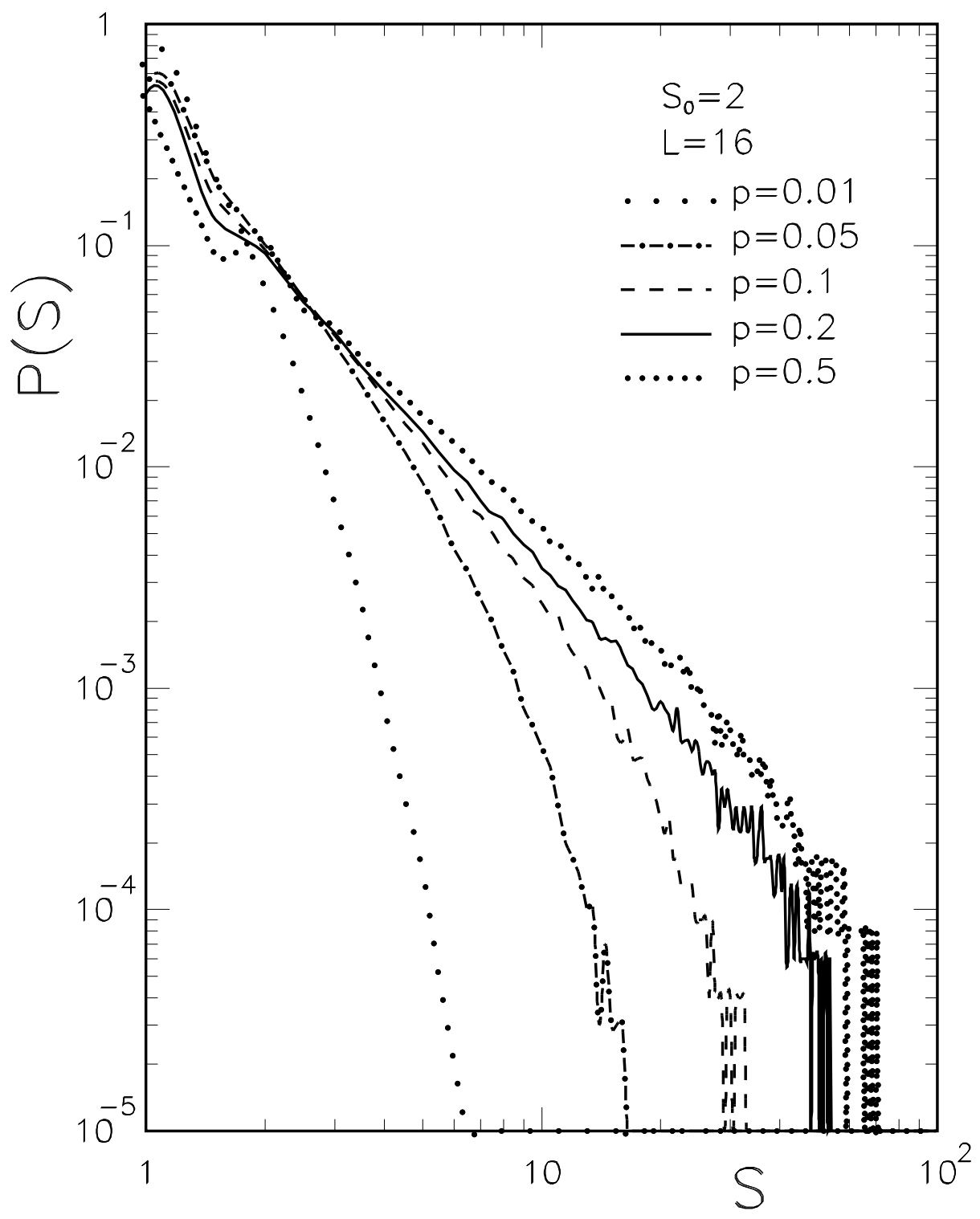

Fig. 5 


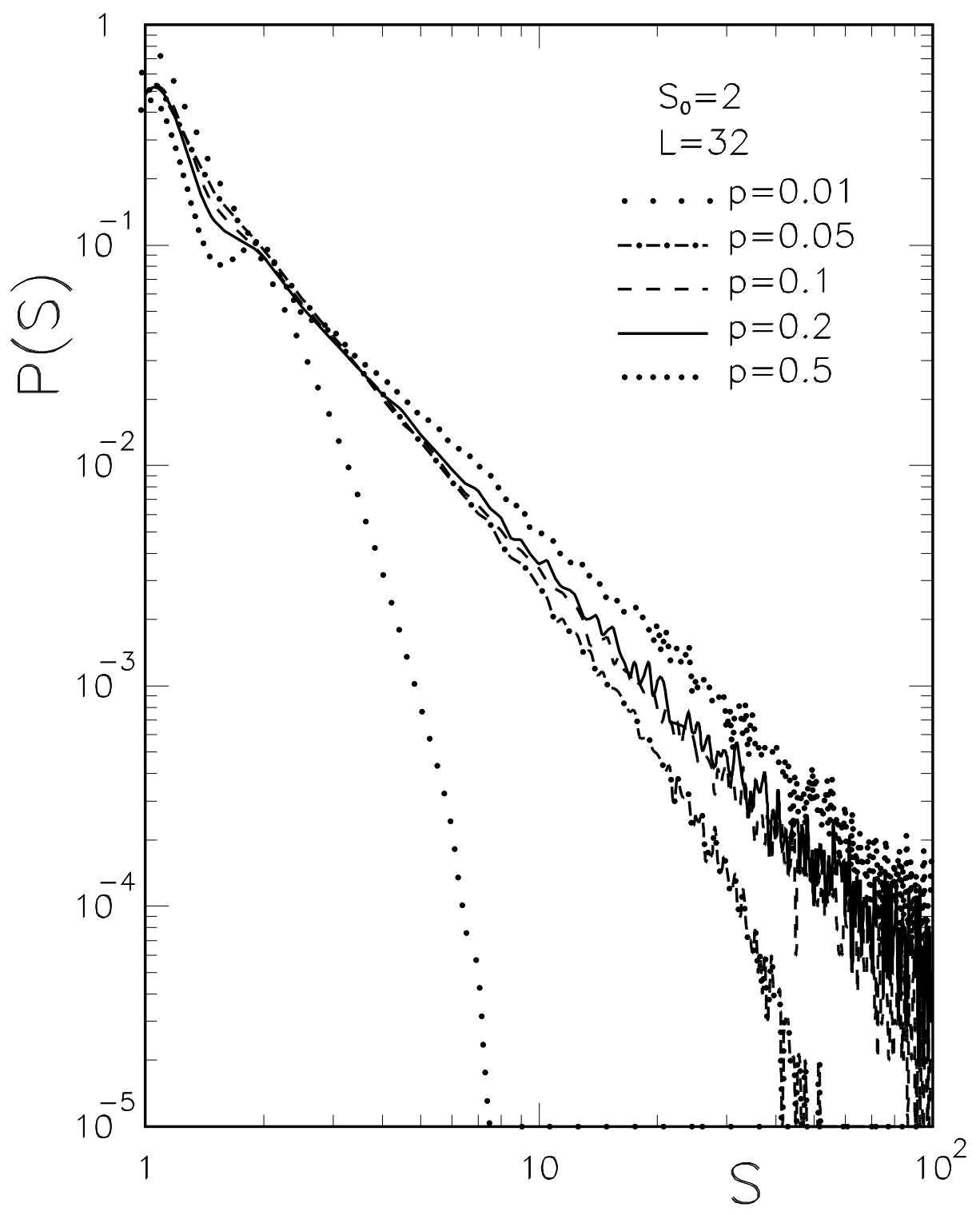

Fig. 6 


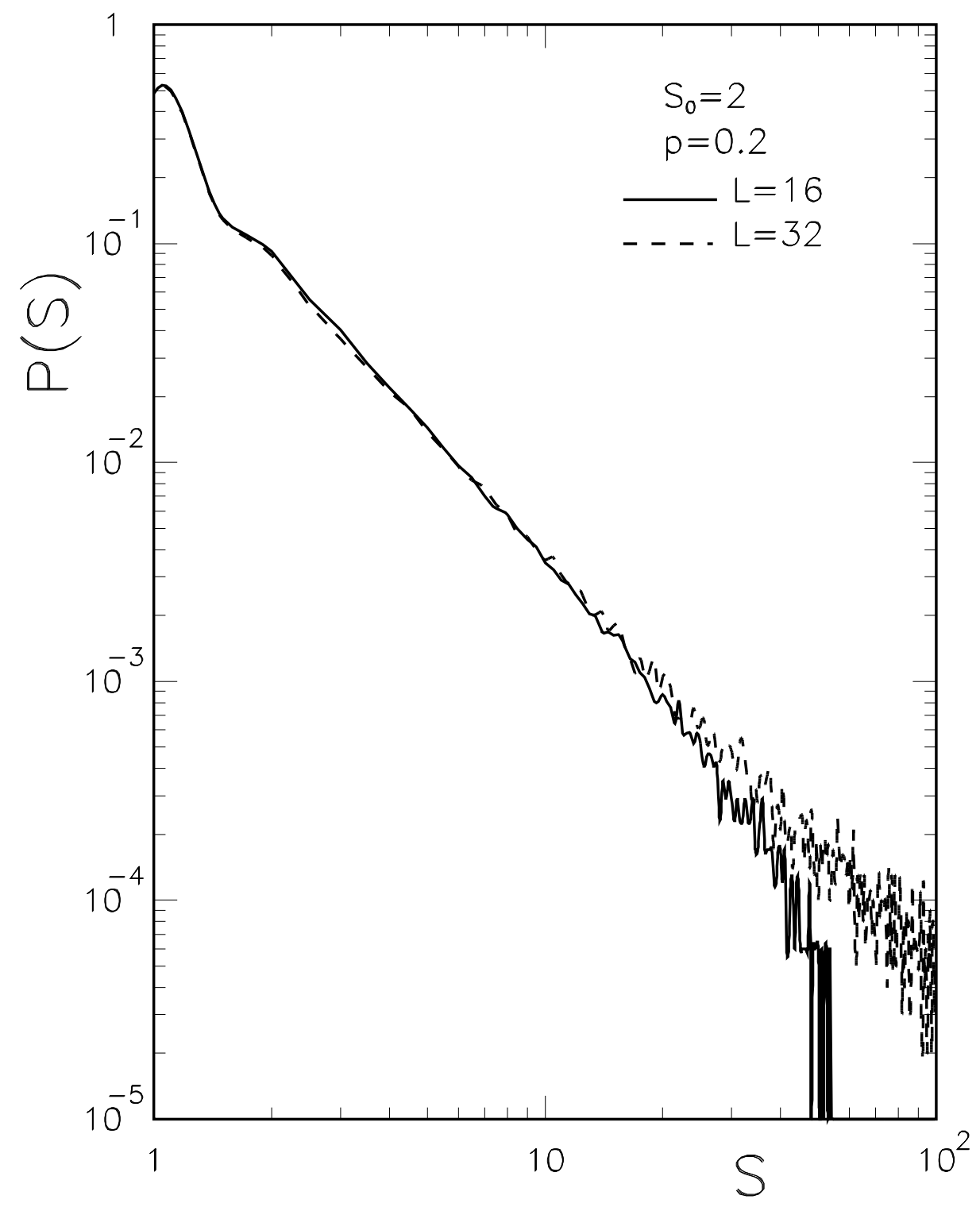

Fig. 7 


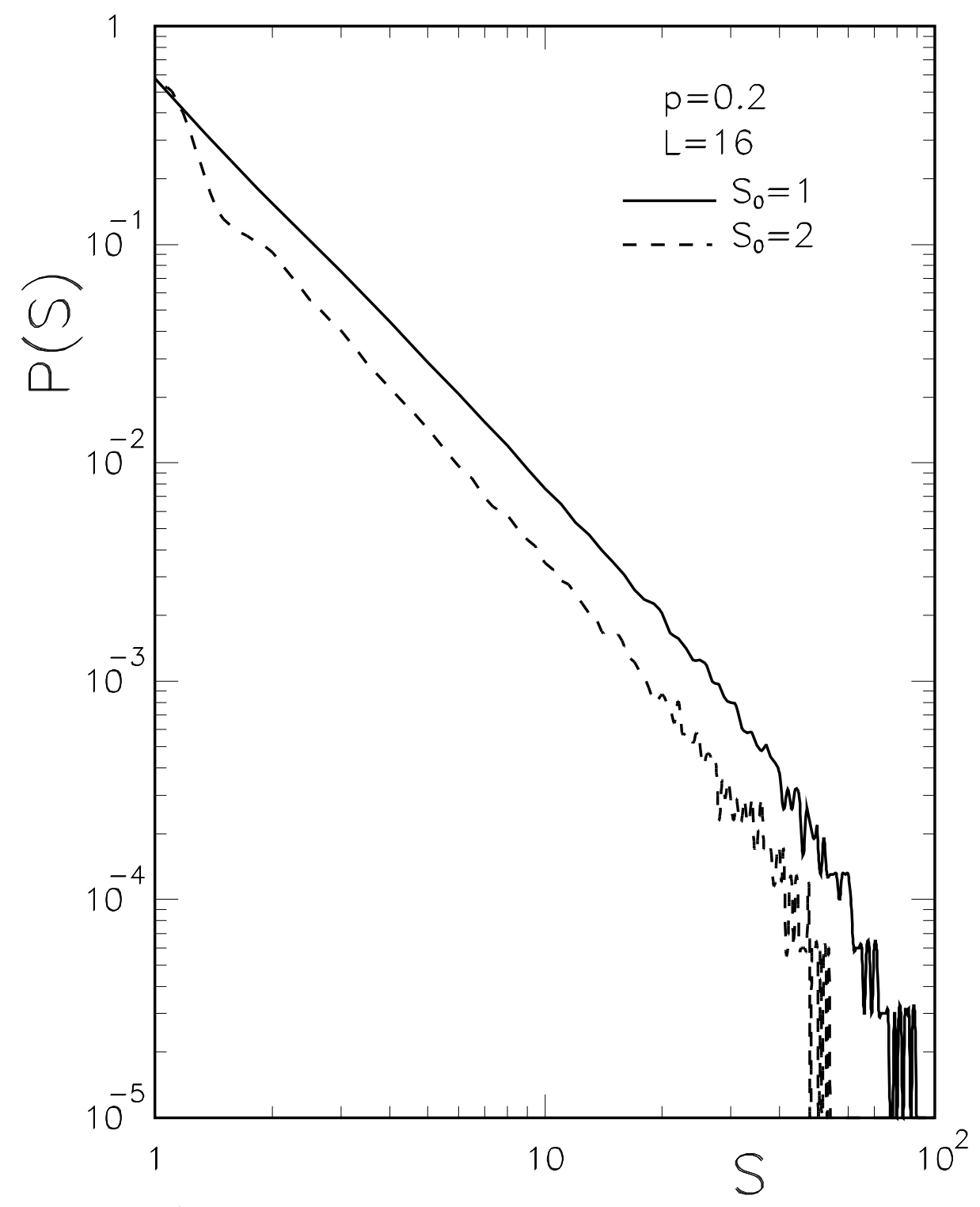

Fig. 8 


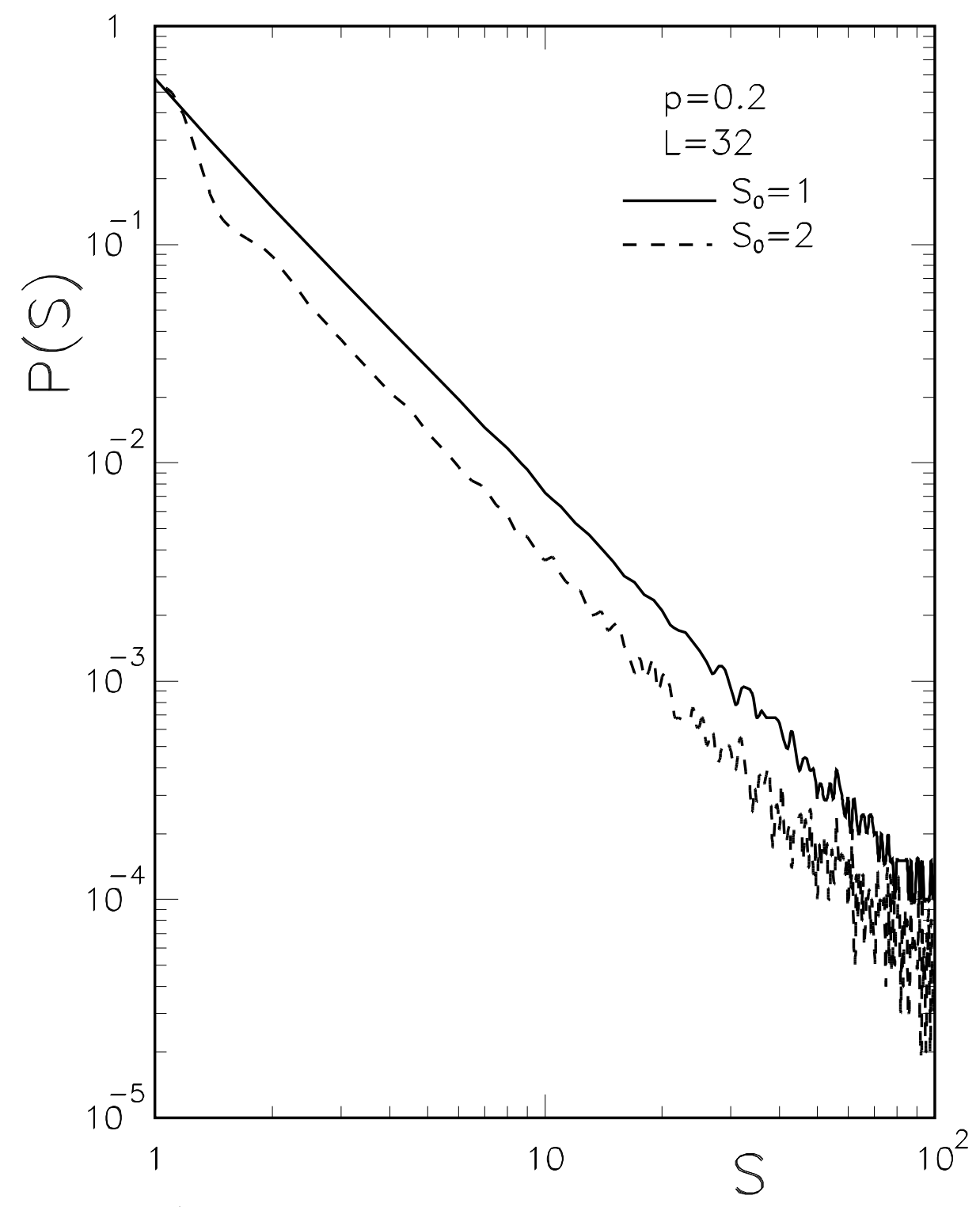

Fig. 9 